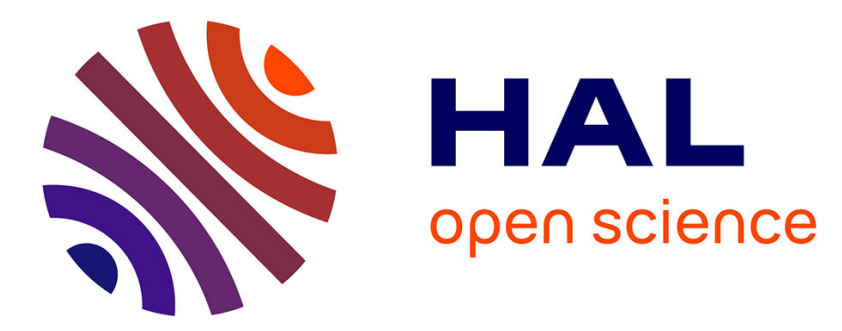

\title{
Unravelling the formation of BAB block copolymer assemblies during PISA in water
}

Pauline Biais, Olivier Colombani, Laurent Bouteiller, François Stoffelbach, Jutta Rieger

\section{> To cite this version:}

Pauline Biais, Olivier Colombani, Laurent Bouteiller, François Stoffelbach, Jutta Rieger. Unravelling the formation of BAB block copolymer assemblies during PISA in water. Polymer Chemistry, 2020, 11 (28), pp.4568-4578. 10.1039/D0PY00422G . hal-02917168

\section{HAL Id: hal-02917168 \\ https://hal.science/hal-02917168}

Submitted on 18 Sep 2020

HAL is a multi-disciplinary open access archive for the deposit and dissemination of scientific research documents, whether they are published or not. The documents may come from teaching and research institutions in France or abroad, or from public or private research centers.
L'archive ouverte pluridisciplinaire HAL, est destinée au dépôt et à la diffusion de documents scientifiques de niveau recherche, publiés ou non, émanant des établissements d'enseignement et de recherche français ou étrangers, des laboratoires publics ou privés. 


\section{Unravelling the formation of BAB block copolymer assemblies during PISA in water}

Pauline Biais $^{\mathrm{a}}$, Olivier Colombani ${ }^{\mathrm{b}, *}$, Laurent Bouteiller ${ }^{\mathrm{a}}$, François Stoffelbach ${ }^{\mathrm{a}}$, Jutta Rieger, ${ }^{\mathrm{a}, *}$

${ }^{a}$ Sorbonne Université, CNRS, Institut Parisien de Chimie Moléculaire, UMR 8232, Polymer

Chemistry Team, 4 Place Jussieu, 75252 Paris Cedex 05, France

E-mail: jutta.rieger@sorbonne-universite.fr

${ }^{\mathrm{b}}$ Institut des Molécules et Matériaux du Mans (IMMM), UMR 6283 CNRS Le Mans Université, Avenue Olivier Messiaen, 72085 Le Mans Cedex 9, France

E-mail: olivier.colombani@univ-lemans.fr

\section{FIGURE FOR TOC}

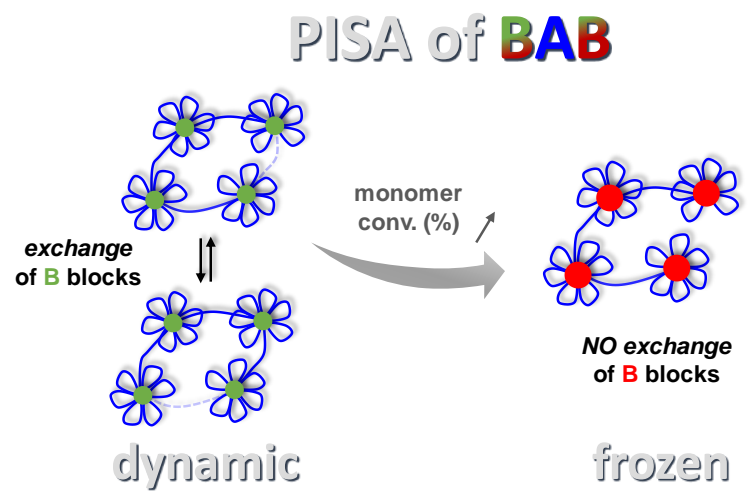

\section{Text for TOC}

BAB triblock copolymers prepared using PISA via aqueous RAFT dispersion polymerization selfassemble into a transient network of bridged micelles. The slowdown of the exchange of B blocks between micellar cores during the polymerization is highlighted as well as the parameters affecting the polymerization. 


\begin{abstract}
:
The mechanism of formation of associative BAB triblock copolymers through aqueous polymerization-induced self-assembly (PISA) is investigated for the first time, on copolymers constituted of a hydrophilic poly $(N, N$-dimethylacrylamide) block (PDMAc = block $\mathrm{A})$ and a hydrophobic poly(diacetone acrylamide) block (PDAAm = block B). Such BAB copolymers tend to form bridged micelles/networks, which was expected to make the PISA process much more complex than for conventional diblock copolymers. Kinetic monitoring, light scattering analyses and macroscopic observations allowed identifying crucial parameters that influence the polymerization and the final dispersion properties, notably the stirring of the polymerization medium, the macroRAFT agent concentration, its ionization degree (related to the $\mathrm{pH}$ ) and its $\mathrm{Z}$ group alkyl chain length.
\end{abstract}

\title{
Introduction:
}

BAB copolymers are amphiphilic triblock copolymers, where the inner A block is hydrophilic and covalently connected to two outer B blocks that are hydrophobic. Such copolymers may find applications as thermoplastic elastomers ${ }^{1}$, filtration membranes ${ }^{2}$ or hydrogels ${ }^{3,4}$ for biomedical applications. They are usually synthesized in solution in a non-selective organic solvent in which both types of blocks are soluble. However, this strategy requires the use of organic solvents and does not allow the formation of amphiphilic triblock copolymers for which no common solvent can be found.

An attractive pathway for the synthesis of amphiphilic block copolymers is polymerization-induced self-assembly (PISA), ${ }^{5-8}$ which consists in forming the B blocks by chain extension of the A block in a selective solvent for the latter, so that polymerization and self-assembly occur simultaneously. PISA can be achieved in water, a green solvent, and may be applied even if the A and B blocks cannot be dissolved in a common non-selective solvent thereby circumventing the limitations encountered in homogeneous solution. In addition, PISA allows the production of amphiphilic block copolymers in high solids contents (up to $50 \mathrm{wt} \%$ ) and is scalable to industrial quantities. In contrast to $\mathrm{AB}$ and $\mathrm{ABA}$ amphiphilic copolymers, only very few BAB triblocks prepared by PISA can be found in the literature. Zhang's group pioneered this field in 2014 and reported the possibility 
to synthesize linear BAB triblock copolymer assemblies through a RAFT dispersion PISA process of styrene conducted in alcohol/water mixtures. ${ }^{9-12}$ In more detail, depending on the macroRAFT agent used, spherical particles, complex aggregates or higher order morphologies (and their aggregates) were observed. ${ }^{9,11}$ Recently, our group reported the first successful synthesis of BAB copolymers by PISA in pure water ${ }^{13}$ : symmetrical bifunctional poly $(N, N$-dimethylacrylamide $)$ (PDMAc) macroRAFT agents with a central benzoic acid group and two outer alkyl chains as Zgroups were used in the aqueous dispersion polymerization of diacetone acrylamide (DAAm) (see structure in Scheme S1). The study notably highlighted the crucial role of the central benzoic acid group in the stabilization of the nano-objects and also revealed an impact of the length of the alkyl chains ends. The scarcity of the examples found in the literature and the problems of colloidal stability and control of the polymerization reported show that the synthesis of BAB triblock copolymers by PISA is much more complex than PISA of AB diblock copolymers and hardly understood. ${ }^{14-17}$

The self-assembly of $\mathrm{BAB}$ triblock copolymers in aqueous medium is well understood when thermodynamic equilibrium can be reached. ${ }^{18,19}$ As for AB diblock copolymers, the B blocks of $\mathrm{BAB}$ copolymers self-assemble in aqueous medium in order to shield themselves from water, leading to spherical hydrophobic cores in the simplest and most frequent case. In dilute solution, the two B blocks of one BAB triblock gather into the same hydrophobic core, so that the A block forms a loop, leading to flower-like micelles (see Scheme 1). At higher concentration, the two B blocks of a single $\mathrm{BAB}$ triblock can enter different hydrophobic cores, bridging flower-like micelles into larger and larger aggregates until a 3D-spanning physical network is eventually formed above a critical percolation concentration $\mathrm{C}_{\mathrm{p}}$.

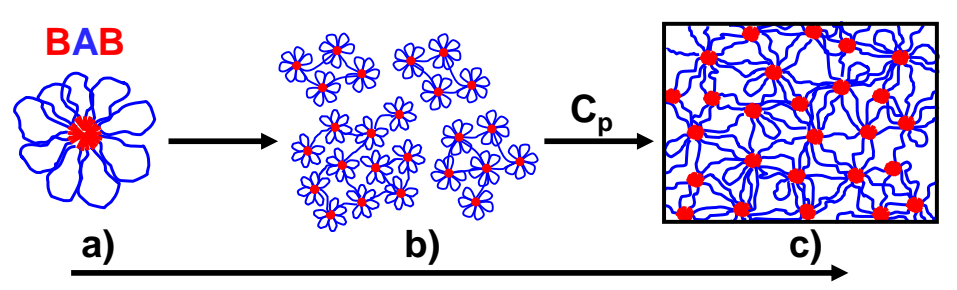

increasing polymer concentration

Scheme 1. Self-assembly of BAB triblocks under thermodynamic equilibrium into a) individual flower-like micelles at low concentration, b) finite size aggregates of connected flower-like 
micelles at intermediate concentration and c) 3D-spanning transient network of connected flowerlike micelles above the percolation concentration.

Attraction caused by the bridges may result in macroscopic phase separation between a very dilute aqueous phase and a concentrated transient percolated network where intermicellar bridges are favored over intramicellar loops. Phase separation is disfavored for charged polymers at low ionic strength (because the entropic penalty is high due to the confinement of the counter-ions in the concentrated polymer phase). In contrast phase separation happens more readily for neutral BAB copolymers or for charged $\mathrm{BAB}$ copolymers at high ionic strength. ${ }^{20-23}$ It is also disfavored when $\mathrm{AB}$ diblock copolymers are added to the $\mathrm{BAB}$ triblock copolymers because this reduces attraction. ${ }^{24}$ The rheological properties of the transient network are controlled by the life-time of the bridges which remains reasonably short (allowing exchange of bridges/B blocks between different hydrophobic cores) if thermodynamic equilibrium is indeed reached.

However, many amphiphilic block copolymers actually form frozen structures in aqueous medium ${ }^{25}$, meaning that the exchange of $\mathrm{B}$ blocks between hydrophobic cores is kinetically hindered within the time scale of observation (because of glassy B blocks and/or because of too hydrophobic B blocks). In that case, self-assembly is impacted by the process and may strongly differ from the scenario described above, where thermodynamic equilibrium is reached. In the case of PISA, the increase of the B block length during polymerization affects self-assembly both in terms of aggregation ${ }^{14}$ and exchange dynamics. Longer B blocks indeed favor aggregation and slow down exchange between cores. ${ }^{7,25,26}$ Moreover, the A block may be pre-assembled if the RAFT agent is functionalized by a long alkyl chain. ${ }^{27}$ Very different organizations at the nanoscale may thus be expected during the synthesis of BAB triblock copolymers by PISA in aqueous medium depending on the initial organization of the bifunctional macroRAFT agent (A block) and on the way the exchange rate of the B blocks and their extent of aggregation are affected by their growth. It is also possible that chain extension of the macroRAFT agent does not happen symmetrically so that the B block on one side reaches a frozen self-assembled state before the other one is large enough to self-assemble, further affecting the behavior of the system. Moreover, if macroscopic phase separation does occur during PISA due to the attraction caused by the bridges, polymerization control may be lost. 
We observed in our former PISA study on PDAAm- $b$-PDMAc- $b$-PDAAm particles, ${ }^{13}$ that changing the initial concentration of macroRAFT agent, the ionization degree of the central benzoic acid function on the R group of the macroRAFT agent or the length of its terminal alkyl chains $\left(\mathrm{C}_{4}\right.$ or $\mathrm{C}_{12}$ ) on its $\mathrm{Z}$ groups (see Scheme 2 ) could result in the loss of colloidal stability during PISA, and was in some cases accompanied by a loss of control over the polymerization. In contrast, suspensions that remained colloidally stable always resulted in controlled polymerizations. In order to better understand the uninvestigated and obviously complex mechanism of self-assembly of BAB triblock copolymers during PISA, in the present study, we monitored a typical synthesis of BAB triblock copolymers by PISA (Scheme 2) and investigated their self-assembly by static and dynamic light scattering as well as qualitative macroscopic observations.

\section{Experimental part:}

\section{Materials}

1,3,5-Trioxane (99\%, Aldrich), 4,4'-azobis(4-cyanopentanoic acid) (ACPA, $\geq 98 \%$ Aldrich) and diacetoneacrylamide (DAAm, 99\%, Aldrich) were used as received.

\section{Polymer synthesis}

The synthesis of the macroRAFT agents $\left(\mathrm{C}_{12} \text {-TTC-PDMAc) }\right)_{2}-\mathrm{BA}$ and $\left(\mathrm{C}_{4} \text {-TTC-PDMAc }\right)_{2}$-BA (named respectively in this study $\mathrm{C}_{12}$-macroRAFT agent and $\mathrm{C}_{4}$-macroRAFT agent) were described in a former study ${ }^{13}$ and detailed in the SI (Scheme S1, Table S1, Figure S1). The macroRAFT agent extensions with DAAm (Scheme 2) conducted with stirring were performed according to a formerly developed protoco ${ }^{13}$ at $70^{\circ} \mathrm{C}$, in a $2 \mathrm{~mL}$ round bottom flask using an oliveshaped stirring bar $(5 \times 10 \mathrm{~mm})$ and a stirring speed of $500 \mathrm{rpm}$ as detailed in the SI (Table S2, Figure S2). The macroRAFT agent extensions with DAAm conducted without stirring during the polymerization as well as the kinetic study performed in $\mathrm{D}_{2} \mathrm{O}$ in NMR tubes are described in details in the SI, section 2.

\section{Sample preparation for static and dynamic light scattering (SLS and DLS)}

The macroRAFT agent powders were dissolved and the copolymer dispersions were diluted in pure milli-Q water at room temperature at the desired concentration. After complete 
dissolution/dispersion, $\mathrm{NaCl}$ concentration was adjusted to $0.01 \mathrm{~mol} \mathrm{~L}^{-1}$ using concentrated stock solutions to prevent any polyelectrolyte effect ${ }^{28}$. For the macroRAFT agent samples, the desired ionization degree was then set by addition of concentrated $\mathrm{NaOH}$ solution. The ionization degree corresponds to the percentage of charged benzoic acid units: $\alpha=\left[\mathrm{COO}^{-}\right] /\left(\left[\mathrm{COO}^{-}\right]+[\mathrm{COOH}]\right)=$ $\mathrm{n}_{\mathrm{NaOH}} / \mathrm{n}_{\text {benzoic acid. }}$ Before analysis, the samples were filtered through $0.45 \mu \mathrm{m}$ pore size filters (except for $\mathrm{C}>20 \mathrm{~g} \mathrm{~L}^{-1}$ for which the solutions were analyzed without filtration). The $\mathrm{NaCl}$ concentration was kept low and the solutions were studied at concentrations were they remained transparent to avoid multiple scattering which would have been detrimental to the data interpretation.

\section{Characterization techniques}

${ }^{1} H$ NMR. DAAm conversion was determined by ${ }^{1} \mathrm{H}$ NMR in $\mathrm{D}_{2} \mathrm{O}$ at room temperature with a Bruker 300 or $400 \mathrm{MHz}$ spectrometer in 5-mm diameter tubes by the relative integration of the protons of the 1,3,5-trioxane at 5.2 ppm and the vinylic protons of DAAm at 6.3-6.2 and 5.7 ppm. $S E C$. Size exclusion chromatography (SEC) analyses of the polymers were carried out with two PSS GRAM $1000 \AA$ columns $\left(8 \times 300 \mathrm{~mm}\right.$; separation limits: 1 to $\left.1000 \mathrm{~kg} \mathrm{~mol}^{-1}\right)$ and one PSS GRAM $30 \AA$ A column $\left(8 \times 300 \mathrm{~mm}\right.$; separation limits: 0.1 to $\left.10 \mathrm{~kg} \mathrm{~mol}^{-1}\right)$ coupled with a differential refractive index (RI) detector and a $\mathrm{UV}$ detector. $\mathrm{DMF}\left(+\mathrm{LiBr}, 1 \mathrm{~g} \mathrm{~L}^{-1}\right)$ at $60{ }^{\circ} \mathrm{C}$ was used as the mobile phase with a flow rate of $0.8 \mathrm{~mL} \mathrm{~min}^{-1}$. Samples were filtrated on $0.2 \mu \mathrm{m}$ pore-size membrane before injection. The SEC analysis of the RAFT agent $\left(\mathrm{C}_{12}-\mathrm{TTC}\right)_{2}$-BA was carried out with two columns thermostated at $40{ }^{\circ} \mathrm{C}$ (PSS SDV, linear M, $8 \mathrm{~mm} \times 300 \mathrm{~mm}$, bead diameter: 5 $\mu \mathrm{m})$, coupled with a differential refractive index (RI) detector and a UV detector. THF at $40{ }^{\circ} \mathrm{C}$ was used as the mobile phase with a flow rate of $1 \mathrm{~mL} \mathrm{~min}^{-1}$. Samples were filtrated on $0.45 \mu \mathrm{m}$ pore-size membrane before injection. The dispersity $\left(\bigoplus=M_{\mathrm{w}} / M_{\mathrm{n}}\right)$, the number-average molar mass $\left(M_{\mathrm{n}}\right)$, and the weight-average molar mass $\left(M_{\mathrm{w}}\right)$ were calculated from the RI signals by a calibration curve based on PMMA standards with OmniSEC 5.11 software.

Static and Dynamic Light Scattering (SLS and DLS). Light scattering (LS) measurements were done with a standard ALV-CGS3 system equipped with an ALV-5003 multi tau correlator system (ALV GmbH, Germany) with a vertically polarized helium-neon laser with wavelength $\lambda=633$ 
$\mathrm{nm}$ as light source. The measurements were done at $20^{\circ} \mathrm{C}\left(\right.$ or $70{ }^{\circ} \mathrm{C}$ ) over a large range of scattering vectors q varying from ca. $3 \times 10^{-4} \AA^{-1}$ to $3 \times 10^{-3} \AA^{-1}$. $q=(4 \pi \mathrm{n} / \lambda) \times \sin (\theta / 2)$, with $\theta$ the angle of observation and $\mathrm{n}$ the refractive index of the solvent.

Static light scattering (SLS). For SLS, the absolute intensity, $R_{\theta}$ in $\mathrm{cm}^{-1}$, scattered by the polymer was determined according to (Equation 1).

$$
R_{\theta}=\frac{I_{\text {solution }}(\theta)-I_{\text {water }}(\theta)}{I_{\text {toluene }}(\theta)} \times\left(\frac{n_{\text {water }}}{n_{\text {toluene }}}\right)^{2} R_{\text {toluene }} \quad(\text { Equation 1) }
$$

With $I_{\text {solution, }} I_{\text {water }}$ and $I_{\text {toluene }}$ the average intensities scattered, respectively, by the solution, the solvent (water), and the reference (toluene) at angle $\theta ; \mathrm{n}_{\text {water }}=1.333$ and $\mathrm{n}_{\text {toluene }}=1.496$ correspond to the refractive indexes of the solvent and the reference respectively; and $R_{\text {toluene }}=1.35 \times 10^{-5}$ $\mathrm{cm}^{-1}$ the Rayleigh ratio of toluene for a wavelength $\lambda=633 \mathrm{~nm}$.

The apparent molar mass $\left(\mathrm{M}_{\mathrm{app}}\right)$ of the solute was calculated from the Rayleigh ratio of the solution as:

$$
\begin{aligned}
\mathrm{M}_{\mathrm{app}} & =\mathrm{R}_{\theta} /(\mathrm{K} \times \mathrm{C}) \quad(\text { Equation 2) } \\
\text { where } \mathrm{K} & =\left(4 \pi^{2} \mathrm{n}_{\text {solvent }}{ }^{2}\right) \times(\partial \mathrm{n} / \partial \mathrm{C})^{2} /\left(\lambda^{4} . \mathrm{N}_{\mathrm{a}}\right)
\end{aligned}
$$

Here $\partial \mathrm{n} / \partial \mathrm{C}=0.15 \mathrm{~mL} \mathrm{~g}^{-1}$ (value known for PDMAc homopolymer in water ${ }^{29}$ ) is the specific refractive index increment of the solute and $\mathrm{N}_{\mathrm{a}}$ is Avogadro's number. This value will be used for all the copolymers analyzed in this work. When the particles were small compared to $\mathrm{q}^{-1}$ and the solutions were sufficiently diluted so that interactions could be neglected, $\mathbf{M}_{\text {app }}$ corresponded to the weight-average molecular weight of the particles. At high polymer concentrations, $\mathbf{M}_{\mathrm{app}}$ deviates from the real molecular weight of the particles either due to repulsive interactions, which decrease $\mathbf{M}_{\text {app }}$ and that are caused by electrostatic interactions and/or excluded volume interactions, or by attractive interactions, which are mainly caused by bridging between flower-like micelles. ${ }^{20}$ The evolution of $\mathrm{M}_{\mathrm{app}}$ with $\mathrm{C}$ therefore gives precious information about the relative strength of repulsive and attractive interactions between the particles.

Dynamic light scattering (DLS). The normalized electric field autocorrelation functions $\left(\mathrm{g}_{1}(\mathrm{t})\right)$ obtained by DLS measurements were analyzed in terms of a relaxation time $(\tau)$ distribution: 


$$
g_{1}(t)=\int A(\tau) e^{\left(\frac{-t}{\tau}\right)} d \tau \quad(\text { Equation 3) }
$$

The REPES routine ${ }^{30}$ was used to obtain $\mathrm{A}(\tau)$ (the fraction of particles with relaxation time $\tau$ defined as a scattering intensity-weighted amplitude) without assuming a specific shape for the distribution. For most polymer solutions, two populations of scattering particles were observed by DLS, a slow and a fast one. In those cases, it was observed that the slow mode of relaxation corresponded to an apparent hydrodynamic radius $\left(\mathrm{R}_{\mathrm{app}}\right)$ value about 10 times as large as for the fast mode of relaxation; implying that the former mode corresponded to particles having a molecular weight at least a hundred times larger than those of the latter mode assuming that the slow mode corresponded to randomly formed fractal aggregates with a fractal dimension close to 2 (note that denser aggregates would exhibit even higher molecular weights, strengthening the conclusion). As a conclusion, even though the slow mode of relaxation contributed significantly to the DLS and SLS signals because of its large molecular weight, its weight percentage within the sample could be neglected. The SLS and DLS data presented in the manuscript therefore correspond to the contribution of the fast mode of relaxation assuming that the contribution of the slow mode in terms of weight concentration is negligible. A similar behavior has already been observed in the literature for many other self-assembling systems and the same treatment was applied to get rid of the contribution of the large aggregates. ${ }^{31}$ The apparent diffusion coefficient $\mathrm{D}$ was calculated from the average relaxation rate of the fast relaxation mode as $\mathrm{D}=\left\langle\tau^{-1}\right\rangle / \mathrm{q}^{2}$ and is related to $\mathrm{R}_{\mathrm{app}}$ according to $\quad$ (Equation 4.

$$
R_{a p p}=\frac{k_{B} T}{6 \pi \eta D} \quad(\text { Equation } 4)
$$

With $k_{B}$ Boltzmann constant, $T$ the absolute temperature and $\eta$ the viscosity of the solvent at the temperature of the experiment. When the particles were small compared to $\mathrm{q}^{-1}$ and the solutions were sufficiently diluted so that interactions could be neglected, $\mathrm{R}_{\text {app }}$ corresponded to the $\mathrm{z}$-average hydrodynamic radius, $\mathrm{R}_{\mathrm{h}}$.

Cryo-TEM. Cryo-transmission electron microscopy (cryo-TEM) was used to determine the morphology and size of the polymer nanoparticles. The samples were diluted in water to $1 \mathrm{wt} \%$ prior to analysis. According to protocols reported elsewhere, ${ }^{32,33}$ thin liquid films of particle dispersions were prepared at room temperature by depositing $4 \mu \mathrm{L}$ of the diluted sample and successive blotting. They were flash frozen in liquid ethane and observed at $-180{ }^{\circ} \mathrm{C}$ on a JEOL 
JEM-2100 LaB6 microscope operating at $200 \mathrm{kV}$ under low-dose conditions (10 electrons $\AA^{-2} \mathrm{~s}^{-1}$ ). Digital images were recorded on a ultrascan 1000, 2k×2k CCD camera (Gatan, USA).

\section{Results and Discussion}

\section{Overview of the self-assembly throughout the PISA process}

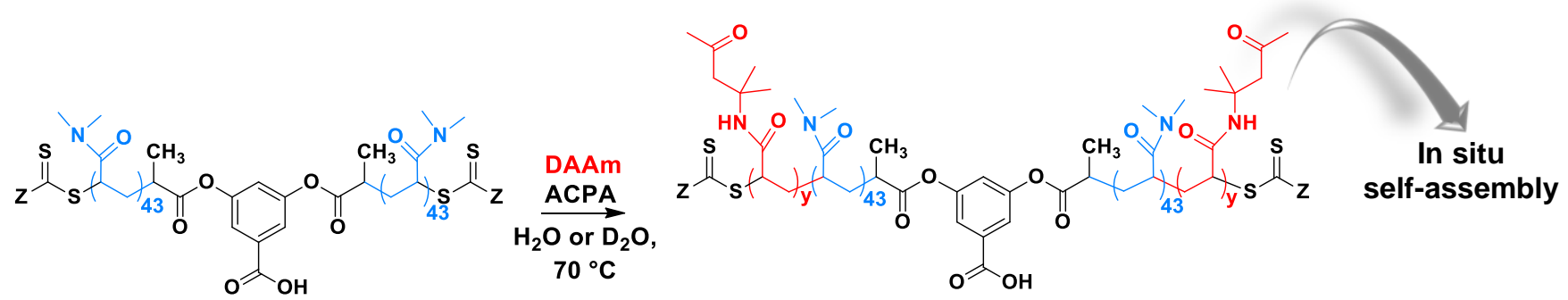

$Z=S-C_{12} H_{25}:\left(C_{12}-T T C-P D M A C\right)_{2}-B A$

$\mathrm{Z}=\mathrm{S}-\mathrm{C}_{4} \mathrm{H}_{9} \quad:\left(\mathrm{C}_{4}-\mathrm{TTC}-\mathrm{PDMAC}\right)_{2}-\mathrm{BA}$

$B_{y} A_{2 x} B_{y}=B A B$ triblock copolymer

$\mathrm{BA}=$ benzoic acid group

Scheme 2. Reaction scheme for the synthesis of PDAAm- $b$-PDMAc- $b$-PDAAm BAB triblock copolymers by polymerization induced self-assembly (PISA) in water.

Stirring may be expected to affect the organization of BAB triblock copolymers prepared by PISA. Indeed, if a percolated network is formed during the process, it might be broken through stirring and will reform only if the B blocks can exchange rapidly compared to the polymerization time. To evaluate the impact of stirring during the dispersion polymerization, PDAAm- $b$-PDMAc- $b$ PDAAm BAB triblock copolymers were synthesized in typical conditions $\left(70{ }^{\circ} \mathrm{C}, 10 \mathrm{wt} \%\right.$ of DAAm), with or without stirring (see Scheme 2, and for the experimental details see SI section 2.a), and Table S2). The RAFT dispersion polymerizations of DAAm were conducted with the previously used ${ }^{13} \mathrm{C}_{12}$-macroRAFT agent $\left(M_{\mathrm{n}, \mathrm{NMR}}=8.6 \mathrm{~kg} \mathrm{~mol}^{-1}\right)$ targeting a $D P_{\mathrm{n} / \mathrm{arm}}$ PDAAm of $\sim 80$ (see entry B and E in Table S2). 


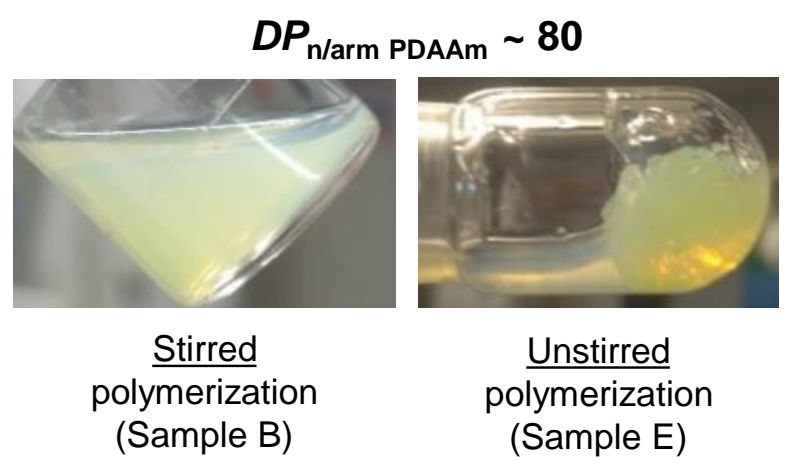

Figure 1. Photographs showing the macroscopic aspect of the final polymerization media synthesized with (left) and without (right) stirring (entry B and E, Table S2).

As shown in the photographs in Figure 1, the absence or presence of stirring during polymerization provided macroscopically very different samples. The polymerization performed under stirring led to a liquid and stable dispersion, whereas the unstirred polymerization phase-separated into a gellike phase and an excluded liquid phase. The resulting gel fraction could not be dispersed again in water, suggesting the formation of a non-dynamic network (see Scheme 1). Interestingly, SEC analysis of the stirred sample and the gel fraction of the unstirred sample (which represents by far the majority of material) revealed a nearly perfect overlay of the SEC chromatograms (see Figure S2) and a relatively narrow molar mass distribution $(\bigoplus<1.3$, see Table S2), demonstrating that the absence of stirring had no significant impact on the control of the polymerization, at least not in these polymerization conditions. In view of these results, we can conclude that the different visual aspect of the samples in the absence or presence of stirring is not related to intrinsic differences in chemistry of the macromolecular chains. It must therefore be correlated to their supramolecular organization and their dynamics during the polymerization.

In order to investigate in more details what happens at the nano-scale during the synthesis of $\mathrm{BAB}$ triblock copolymers by PISA and understand the role of stirring, we used LS to characterize the self-assembly in aqueous medium of the BAB triblock copolymers for different degrees of polymerization of the B blocks (corresponding to different monomer conversions during the PISA process). In our previous study ${ }^{13}$ we had shown that the $\mathrm{pH}$ of the solution, i.e. the ionization degree $(\alpha)$ of the central benzoic acid group had an impact on the assembly. Here, we set $\alpha$ at a constant value of $28 \%$ for a $[\mathrm{DAAm}]_{0} /\left[\mathrm{C}_{12} \text {-macroRAFT }\right]_{0}=100$ (see Table 1), which corresponds to 
conditions where tiny spherical particles $\left(\mathrm{d}_{\text {cryo-TEM }} \sim 20 \mathrm{~nm}\right)$ had been obtained. First the $\mathrm{C}_{12-}$ macroRAFT agent (see Table S1, entry $\mathrm{C}_{12}$-PDMAc-bis $M_{\mathrm{n}, \mathrm{NMR}}=8.5 \mathrm{~kg} \mathrm{~mol}^{-1}$ ) was analyzed by SLS and DLS in water at $20{ }^{\circ} \mathrm{C}$ (see Figure 2). To investigate the self-assembly over a broad range of concentrations (from 0.2 to $70 \mathrm{~g} \mathrm{~L}^{-1}$ ), the macroRAFT agent was dissolved at $70 \mathrm{~g} \mathrm{~L}^{-1}$ and then diluted to the different concentrations.

The evolution of the apparent molar mass $\left(\mathrm{M}_{\mathrm{app}}\right)$ with the polymer concentration corresponded to the typical signature of $\mathrm{BAB}$ triblock copolymers self-assembling under thermodynamic equilibrium. ${ }^{20}$ Indeed, in diluted conditions $\left(\mathrm{C}<2 \mathrm{~g} \mathrm{~L}^{-1}\right), \mathrm{M}_{\mathrm{app}}$ was constant $\left(\mathrm{M}_{\text {app }} \sim 140 \mathrm{~kg} \mathrm{~mol}^{-1}\right)$ and corresponded to the molecular weight of the self-assembled particles. This value gave an aggregation number $\left(\mathrm{N}_{\mathrm{agg}}\right)$ of $14\left(\mathrm{~N}_{\mathrm{agg}}=\mathrm{M}_{\mathrm{app}}\right.$ (particles) $/ M_{\mathrm{w}}\left(\mathrm{C}_{12}\right.$-PDMAc-bis $)$ with $M_{\mathrm{w}}=M_{\mathrm{n}, \mathrm{NMR}}$ $\left(\mathrm{C}_{12}\right.$-PDMAc-bis $\left.) \times Ð=8.5 \times 1.18=10.0 \mathrm{~kg} \mathrm{~mol}^{-1}\right)$. Moreover, the determined hydrodynamic radius $\left(\mathrm{R}_{\mathrm{h}} \sim 7 \mathrm{~nm}\right)$ was compatible with the formation of individual flower-like micelles. ${ }^{\dagger}$ Above $3 \mathrm{~g} \mathrm{~L}^{-1}$, a sharp increase of $\mathrm{M}_{\text {app }}$ with concentration was observed due to attractive interactions, which is typical of the formation aggregates of bridged micelles (see Scheme 1). At even higher concentrations $\left(\mathrm{C}>20 \mathrm{~g} \mathrm{~L}^{-1}\right)$ the repulsive excluded volume interactions dominated, leading to a decrease of $\mathrm{M}_{\mathrm{app}}$.

This study showed that the $\mathrm{C}_{12}$ alkyl chains were hydrophobic enough to trigger self-assembly of the $\mathrm{C}_{12}$-macroRAFT agent. Moreover, the self-assembly was dynamic (at thermodynamic equilibrium) since the observed variations of $\mathbf{M}_{\text {app }}$ with concentration could not have been obtained by successive dilutions from $70 \mathrm{~g} \mathrm{~L}^{-1}$ if the bridges between the flower-like micelles had been frozen. Finally, the evolution of $\mathrm{M}_{\mathrm{app}}$ with concentration and the increase of the viscosity qualitatively observed with the naked eye above $\mathrm{C}=30 \mathrm{~g} \mathrm{~L}^{-1}$ implied that a percolated transient network was formed at $70 \mathrm{~g} \mathrm{~L}^{-1}$, that is the macroRAFT concentration at which the PISA process was performed. The whole behavior of this polymer was consistent with what was reported for telechelic poly(ethylene oxide) decorated on both sides by $\mathrm{C}_{12}$ alkyl chains. ${ }^{21,34}$ The polymerization temperature being $70{ }^{\circ} \mathrm{C}$, we also performed LS of the macroRAFT agent at this temperature, but no significant differences in aggregation were observed when comparing the LS experiments at

\footnotetext{
† The maximum theoretical length of the stretched PDMAc corona is $\mathrm{R}_{\mathrm{h}, \max }=0.25 \times D P_{\mathrm{n} / \mathrm{arm}}$ $\mathrm{PDMAc}=11 \mathrm{~nm}$.
} 
either $20^{\circ} \mathrm{C}$ or $70{ }^{\circ} \mathrm{C}$ (see Figure S3). We could thus reasonably conclude that the polymerization of DAAm started from a dynamic transient percolating network of $\mathrm{C}_{12}$-macroRAFT.

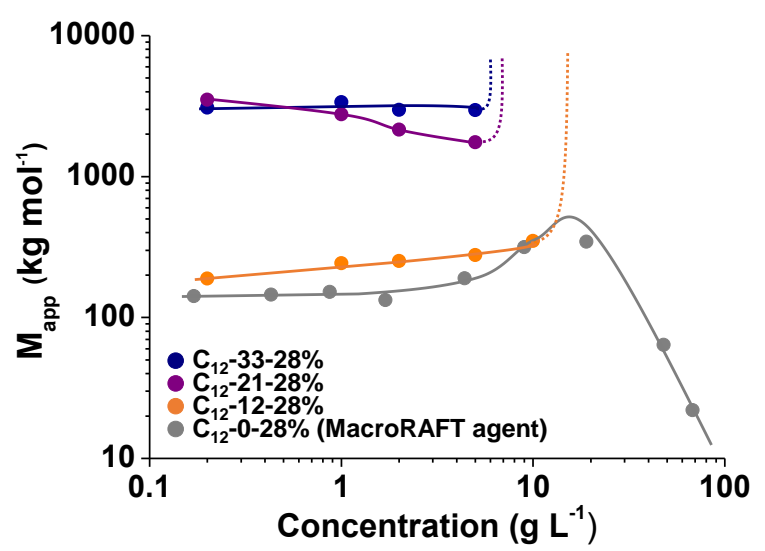

Figure 2. Evolution of the apparent molar mass $\mathrm{M}_{\mathrm{app}}$ as a function of the polymer concentration obtained by static light scattering at $20^{\circ} \mathrm{C}$ in water for the macroRAFT agent $\mathrm{C}_{12}$-PDMAc-bis and for block copolymers with $D P_{\mathrm{n} / \mathrm{arm}}=12,21$ and 33 prepared by dispersion polymerization of DAAm in $\mathrm{D}_{2} \mathrm{O}$ in the presence of the macroRAFT agent $\mathrm{C}_{12}$-PDMAc-tris, at $\alpha=28 \%$, without stirring (see Table 1). The solid lines are guides for the eyes. Dotted lines indicate precipitation of the sample caused by the addition of salt used for LS analyses $\left([\mathrm{NaCl}]=0.01 \mathrm{~mol} \mathrm{~L}^{-1}\right)$.

Thereafter, a typical dispersion polymerization of DAAm in the presence of the $\mathrm{C}_{12}$-macroRAFT agent (see Table S1, entry $\mathrm{C}_{12}$-PDMAc-tris, $M_{\mathrm{n}, \mathrm{NMR}}=8.7 \mathrm{~kg} \mathrm{~mol}^{-1}$ ) was conducted in $\mathrm{D}_{2} \mathrm{O}$, without stirring $\left(70{ }^{\circ} \mathrm{C}, 10 \mathrm{wt} \%\right.$ of DAAm, $\left[\mathrm{C}_{12} \text {-macroRAFT }\right]_{0}=70 \mathrm{~g} \mathrm{~L}^{-1}$, with $\alpha=28 \%$, see experimental details in Table 1 and protocol in SI, part 2.b). The polymerization was stopped at different monomer conversions (corresponding to $D P_{\mathrm{n} / \text { arm PDAAm }}$ of 12, 21, 33 and 49, see Table 1) and the samples were analyzed by SEC and LS. SEC analyses (Figure S4) showed the expected shift of the mass distributions with increasing monomer conversion corroborating the formation of block copolymers. ${ }^{1} \mathrm{H}$ NMR-monitoring of the polymerization in similar conditions $\left(70{ }^{\circ} \mathrm{C}, 10 \mathrm{wt} \%\right.$ of DAAm, $[\mathrm{DAAm}]_{0} /\left[\mathrm{C}_{12} \text {-macroRAFT }\right]_{0}=100$ ) indicated the complete consumption of the $\mathrm{C}_{12}$ macroRAFT agent at low conversions (conv. < 5\% see Figure S5A and corresponding comment in the SI) which indicated that a triblock copolymer was indeed formed at very low monomer conversion. 
Table 1. Kinetic monitoring of the formation of BAB triblock copolymers: unstirred dispersion polymerizations of $\mathrm{DAAm}$ in $\mathrm{D}_{2} \mathrm{O}$ in the presence of the symmetric $\mathrm{C}_{12}-$ macroRAFT agent at $\alpha=28 \%$ and $\left[\mathrm{C}_{12} \text {-macroRAFT }\right]_{0}=70 \mathrm{~g} \mathrm{~L}^{-1} a$

\begin{tabular}{|c|c|c|c|c|c|c|c|}
\hline Entry & $\begin{array}{c}\operatorname{conv}^{b} \\
(\%)\end{array}$ & $\begin{array}{c}\mathbf{C}(\text { polym }) \\
\left(\mathrm{g} \mathrm{L}^{-1}\right)\end{array}$ & $\underset{\text { PDAAm }}{D P_{\mathrm{n} / \mathrm{arm}^{c}}}$ & $\begin{array}{c}M \mathrm{n}, \mathrm{th}^{c} \\
\left(\mathrm{~kg} \mathrm{~mol}^{-1}\right)\end{array}$ & $\begin{array}{l}M_{\mathrm{n}, \mathrm{PMMA}^{d}} \\
\left(\mathrm{~kg} \mathrm{~mol}^{-1}\right)\end{array}$ & $\bigoplus^{d}$ & $\begin{array}{c}\text { Phase } \\
\text { sep. }\end{array}$ \\
\hline $\mathrm{C}_{12-12-28 \%}$ & 25 & 105 & 12 & 12.9 & 9.3 & 1.20 & no \\
\hline $\mathrm{C}_{12-21-28 \%}$ & 42 & 128 & 21 & 15.7 & 11.1 & 1.24 & no \\
\hline $\mathrm{C}_{12}-33-28 \%$ & 66 & 160 & 33 & 19.8 & 13.1 & 1.21 & no \\
\hline $\mathrm{C}_{12-49-28 \%} \%$ & 97 & 193 & 49 & 25.3 & 21.5 & 1.34 & no \\
\hline
\end{tabular}

${ }^{a}$ Polymerization conditions: $\mathrm{T}=70{ }^{\circ} \mathrm{C},[\mathrm{DAAm}]_{0}=10 \mathrm{wt} \%, \alpha(\mathrm{BA})=28 \%, M_{\mathrm{n}, \mathrm{NMR}}\left(\mathrm{C}_{12}\right.$-PDMAc-tris $)=$ $8.7 \mathrm{~kg} \mathrm{~mol}^{-1},\left[\mathrm{C}_{12} \text {-macroRAFT }\right]_{0}=70 \mathrm{~g} \mathrm{~L}^{-1}=8 \mathrm{mmol} \mathrm{L}^{-1}$, $[\mathrm{ACPA}]_{0}=0.9 \mathrm{mmol} \mathrm{L}{ }^{-1} \cdot{ }^{b}$ Monomer conversion determined by ${ }^{1} \mathrm{H}$ NMR. ${ }^{c}$ Theoretical number-average degree of polymerization, $D P_{\mathrm{n} / \mathrm{arm} \text { PDAAm }}$, and numberaverage molar mass, $M_{\mathrm{n}, \mathrm{th}}$, calculated using the experimental conversion and using $M_{\mathrm{n}, \mathrm{NMR}}$ for the PDMAc block (Table S1). ${ }^{d}$ Number-average molar mass and dispersity, $\oslash$, determined by SEC in DMF $(+\mathrm{LiBr} 1 \mathrm{~g}$ $\mathrm{L}^{-1}$ ) with a PMMA calibration. *For experiment $\mathrm{C}_{12}-49-28 \%$ the polymerization was conducted in water without stirring according to the protocol described in our previous work. ${ }^{13}$ Note that the sample names $\mathrm{C}_{12}-$ $\mathrm{X}-28 \%$ stands for a block copolymers of $D P_{\mathrm{n} / \mathrm{arm}}$ PDAAm $=\mathrm{X}$ synthesized at $\alpha=28 \%$ with $\mathrm{C}_{12}$-macroRAFT agent.

Each sample was studied by LS following the same procedure: the concentrated sample withdrawn from the PISA reaction was diluted to different concentrations before measurement. For DP $\mathrm{n}_{\mathrm{arm}}$ PDAAm $=12$ (25\% monomer conversion), the values of $\mathrm{M}_{\mathrm{app}}$ and $\mathrm{R}_{\mathrm{h} \text {,app }}$ at low concentration were only slightly higher than those determined for the macroRAFT agent and similar $\mathrm{N}_{\text {agg }}$ were determined (see Figure S6). This suggests that similar bridged flower-like micelles were obtained $\left(\mathrm{M}_{\mathrm{app}}=200 \mathrm{~kg} \mathrm{~mol}^{-1}\right.$ corresponding to $\mathrm{N}_{\mathrm{agg}} \sim 13, \mathrm{R}_{\mathrm{h}, \mathrm{app}}=10 \mathrm{~nm}$ which was still smaller than $\mathrm{R}_{\mathrm{h}, \max }$ $=0.25 \times D P_{\mathrm{n} / \mathrm{arm}}=13 \mathrm{~nm}$ ), see Figure 2. It was not possible to investigate the sample at $\mathrm{C} \geq 15 \mathrm{~g}$ $\mathrm{L}^{-1}$ by LS, because the solutions were turbid in the conditions of the $\mathrm{LS}$ analysis $([\mathrm{NaCl}]=0.01$ mol L $\mathrm{L}^{-1}$ added before LS measurement), suggesting phase separation due to strong attraction (bridging of flower-like micelles). Note that no turbidity was observed in the polymerization medium for such concentration. This shows that the addition of a small amount of salt was responsible for the turbidity; probably because the presence of salt favors phase separation ${ }^{20}$ as mentioned in the introduction (see experimental part and Figure S7 for details). At higher monomer conversions, when the length of the hydrophobic block increased to $D P_{\mathrm{n} / \text { arm PDAAm }}=21$ or 33, dilution of the concentrated solutions of the BAB copolymers to $\mathrm{C}<5 \mathrm{~g} \mathrm{~L}^{-1}$ produced much larger objects with a $\mathrm{M}_{\mathrm{app}}$ of $\sim 3.0 \times 10^{3} \mathrm{~kg} \mathrm{~mol}^{-1}$ (corresponding to $\mathrm{N}_{\mathrm{agg}} \sim 130$ ) and a $\mathrm{R}_{\mathrm{h}, \mathrm{app}}$ of $\sim$ 
$35 \mathrm{~nm}$ (see Figure 2). These large values, and especially the fact that $\mathrm{R}_{\mathrm{h} \text {,app }}$ exceeded by far the maximum length expected for fully stretched blocks $\left(\mathrm{R}_{\mathrm{h}, \max }=0.25 \times D P_{\mathrm{n} / \mathrm{arm}} \sim 15 \mathrm{~nm}\right)$ suggested that dilution of the copolymers did not yield individual flower-like micelles but tiny aggregates of flower-like micelles. The fact that the aggregates of flower-like micelles were not disassembled into individual flower-like micelles even at $0.2 \mathrm{~g} \mathrm{~L}^{-1}$ hinted at the formation of frozen structures unable to reorganize upon dilution. The final sample obtained at almost quantitative monomer conversion $(97 \%)$ with the longest PDAAm B-blocks $\left(D P_{\text {n/arm PDAAm }}=49\right)$ was a macroscopic gel that could not be dispersed in water afterwards, confirming that a non-dynamic percolated network was obtained for this final $D P_{\mathrm{n}}$.

From this series of experiments, we concluded that the PISA polymerization of DAAm with $\left[\mathrm{C}_{12}\right.$ macroRAFT] $\sim 70 \mathrm{~g} \mathrm{~L}^{-1}$ and $\alpha=28 \%$ starts in a percolated network of bridged spherical flowerlike micelles (see Scheme 3). Initially, the exchange of B blocks (which are only $\mathrm{C}_{12}$ alkyl chains at $0 \%$ conversion) between hydrophobic cores was quite dynamic. However, with the increase of the length of the B blocks during polymerization the exchange rate slowed down. Indeed, the B blocks still obviously exchanged rapidly between hydrophobic cores (at least within the time scale of the dilution of the samples, $<30 \mathrm{~min}$ ) for $D P_{\mathrm{n} / \mathrm{arm} \text { PDAAm }}=12$, whereas they were certainly frozen for $D P_{\mathrm{n} / \mathrm{arm} \text { PDAAm }}=49$. The transition between a dynamic and a frozen network probably occurred between $D P_{\text {n/arm PDAAm }}=12$ and 21 according to the LS results. ${ }^{\ddagger}$ This behavior explains the differences between the syntheses performed with or without stirring: the network which was present from the beginning of the reaction remained intact throughout the reaction in the absence of stirring; on the contrary, stirring broke the network into finite size aggregates of flower-like micelles and the latter could no more reconnect into a macroscopic gel once the exchange of $\mathrm{B}$ blocks was too slow (see Scheme 3). This latter phenomenon explains why cryo-TEM and LS analyses on representative BAB dispersions synthesized with stirring (see Table $\mathbf{S 2}$, entry C) still revealed the formation of tiny individual particles, which are most probably small aggregates of a few connected flower-like micelles (see Scheme 3, Figure S8 and comments to the figure in the SI).

\footnotetext{
* We note that the conversion is not total for these $D P_{\mathrm{n}}$. Residual monomer may swell the hydrophobic core and thereby impact the exchange dynamics of the B blocks. This would shift the $D P_{\mathrm{n}}$-range where a transition between fast and slow exchange occurs towards higher $D P_{\mathrm{n}}$ values. However, the qualitative conclusion that increasing monomer conversion slows down the exchange dynamics remains valid.
} 


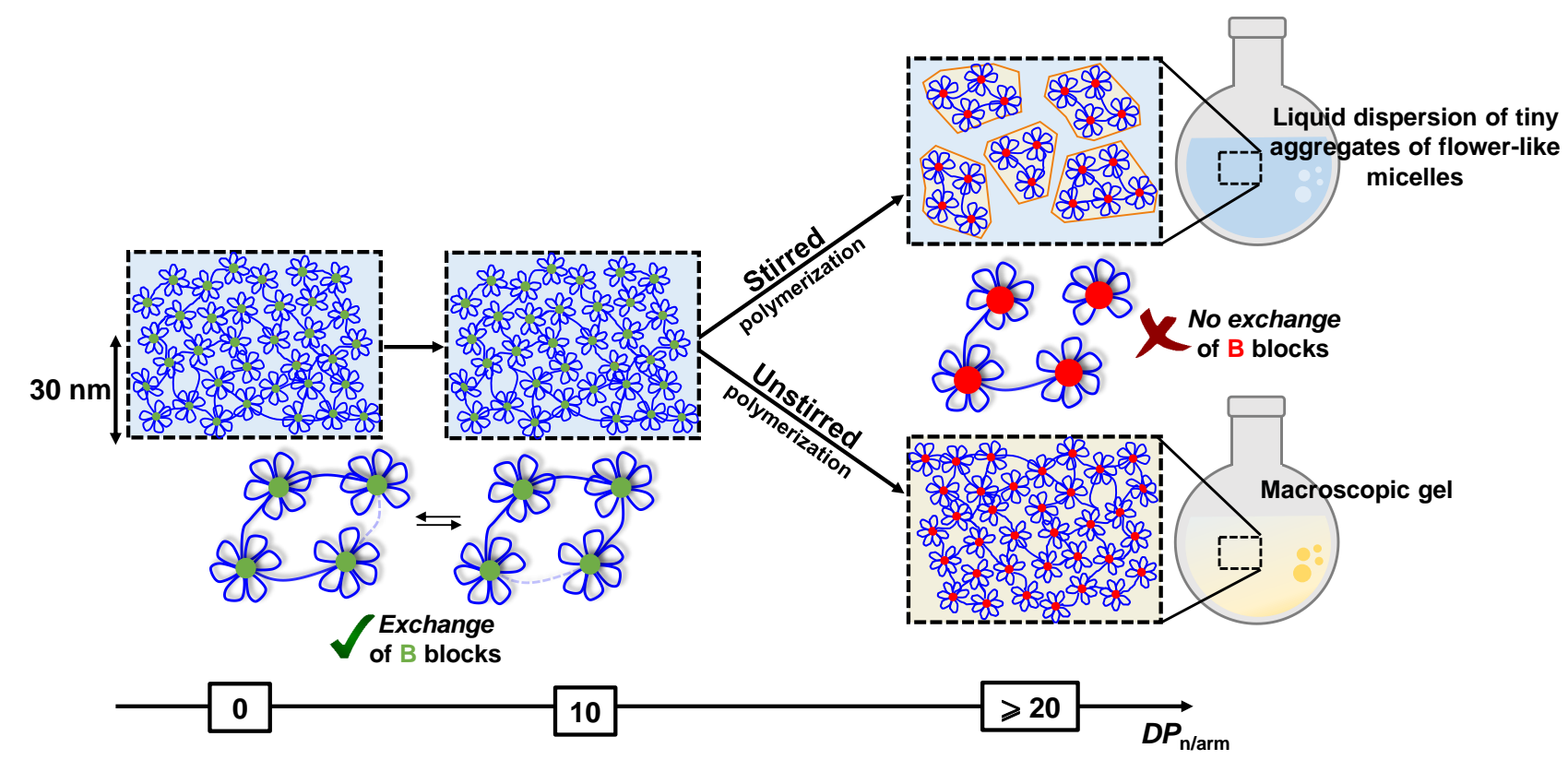

Scheme 3. Schematic representation of the aggregates/network formed with and without stirring during the synthesis of BAB copolymers by PISA.

\section{Parameters influencing phase separation and their consequence on polymerization control}

In the previous section, we showed that PISA starts in a percolating transient network of $\mathrm{C}_{12}$ macroRAFT agent that gradually becomes frozen during polymerization. In some cases (see for example sample $\mathrm{E}$ on Figure 1 at $\left[\mathrm{C}_{12} \text {-macroRAFT }\right]_{0}=33 \mathrm{~g} \mathrm{~L}^{-1}$ and $\alpha=28 \%$ ), this was accompanied with a phase separation. We therefore explored in more details the influence of macroRAFT agent concentration, $\alpha$ and the length of the alkyl chain on phase separation in order to understand its origin and determine its role on the control of the polymerization. We stress that understanding these aspects is of outmost importance for the efficient design of BAB triblock copolymers by PISA, both in terms of colloidal stability and control of the polymerization.

\subsection{Role of the macroRAFT agent concentration and ionization degree ( $\alpha)$}

The role of the macroRAFT agent concentration was investigated first. In an additional series of unstirred experiments, we performed polymerizations at $70{ }^{\circ} \mathrm{C}$, at constant monomer concentration 
(10 wt\% of DAAm), varying the initial concentration of macroRAFT agent (see Table S2, entry $\mathrm{D}, \mathrm{E}$ and F) but keeping constant the ionization degree of the central benzoic acid moiety ( $\alpha=28 \%$ ) and the final monomer conversion $(\sim 95 \%)$. These conditions corresponded to targeted $D P_{\mathrm{n} / \mathrm{arm}}$ PDAAm of 49, 79 and 140 (for $\left[\mathrm{C}_{12} \text {-macroRAFT] }\right]_{0} \sim 67,33$ and $20 \mathrm{~g} \mathrm{~L}^{-1}$, respectively) at full monomer conversion. Macroscopically, an obvious phase separation into a non-dispersible gel phase and a liquid phase was observed with the two lowest macroRAFT agent concentrations (see $\left[\mathrm{C}_{12} \text {-macroRAFT }\right]_{0} \sim 33$ and $20 \mathrm{~g} \mathrm{~L}^{-1}$ in Figure S9i). The volume fraction of the liquid phase increased with decreasing macroRAFT concentration and therefore with increasing length of the PDAAm arms. In contrast, it has been reported in the literature that $\mathrm{AB}^{35}$ and $\mathrm{ABA}^{36}$ PDMAc/PDAAm block copolymers prepared by PISA in water (with block lengths comparable to those in our study) led to stable dispersions. Furthermore, Zhang et al. compared AB diblock copolymers with BAB triblock copolymers prepared by PISA in a mixture of alcohol and water: whereas the former resulted in stable dispersions, the BAB system resulted in phase separation. ${ }^{9}$ This corroborates that the observed phase separation for BAB copolymer dispersions is certainly caused by their specific associative behavior. ${ }^{18}$ Phase separation indeed increases the proportion of intermicellar bridges over intramicellar loops in the polymer-rich phase; which increases the conformational entropy of the B blocks and is favorable. The higher the propensity of the BAB triblocks to form bridges rather than loops (stronger attraction), the more phase separation will be favored. These results indicated qualitatively that the attraction caused by the bridging of micelles was sufficient at $\alpha=28 \%$ to trigger phase separation for $\left[\mathrm{C}_{12} \text {-macroRAFT }\right]_{0} \leq 33 \mathrm{~g} \mathrm{~L}^{-1}$, corresponding - in the experimental conditions - to a $D P_{\mathrm{n} / \mathrm{arm}}$ PDAAm $\geq 79$. At the molecular level, SEC indicated that the polymerization control was maintained in spite of the obvious phase separation for $D P_{\mathrm{n} / \mathrm{arm} \text { PDAAm }}=79$, but not for $D P_{\mathrm{n} / \text { arm PDAAm }}=140$ corresponding to the lowest macroRAFT agent concentration (Figure S9ii). In contrast, we observed that, the synthesis of copolymers with similar $D P_{\mathrm{n} / \text { arm }}$ performed under stirring resulted in a controlled polymerization with no phase separation (see Table S2, entry A, B and C and Figure S10).

Next, the role of $\alpha$ was investigated. In our former study using stirring, ${ }^{13}$ we had observed that colloidal stability and control of the polymerization were lost when the polymerizations were conducted either at a low $\mathrm{pH}$ (corresponding to $\alpha<7 \%$ ) or with a neutral macroRAFT agent (absence of a central benzoic acid moiety in the RAFT agent). As described above, unstirred polymerizations performed with a monomer concentration of $10 \mathrm{wt} \%$ and $\left[\mathrm{C}_{12} \text {-macroRAFT }\right]_{0}=70$ 
$\mathrm{g} \mathrm{L}^{-1}$ (corresponding to $D P_{\mathrm{n} / \mathrm{arm}}$ PDAAm $=49$ at full conversion) were well-controlled and colloidally stable at $\alpha=28 \%$ (see Table 1 and Figure S4). As expected, we obtained similar results when the copolymers were synthesized at a higher ionization degree $(\alpha=45 \%)$ (see Table S3 and Figure S11A).

To understand the role of $\alpha$, its impact on the balance between attraction and repulsion was investigated qualitatively by LS on the $\mathrm{C}_{12}$-macroRAFT agent, at $\alpha=7 \%, 28 \%$ and $78 \%$. As shown in Figure 3, at low concentrations, varying $\alpha$ hardly affected $\mathrm{N}_{\mathrm{agg}}$ of the individual flower-like micelles. However, between 5 and $20 \mathrm{~g} \mathrm{~L}^{-1}, \mathrm{M}_{\text {app }}$ increased much more strongly with concentration for lower $\alpha$. An increase of $\mathrm{M}_{\mathrm{app}}$ with increasing polymer concentration $\mathrm{C}$ indicates that attraction (due to bridging of flower-like micelles) dominates the behavior of the self-assemblies; the steeper the slope, the stronger the effect. Qualitatively, in the Figure 3, the slope in the range 5 to $20 \mathrm{~g} \mathrm{~L}^{-}$ ${ }^{1}$ was greater for lower degree of ionizations $\alpha$, indicating that attraction was strongly increased at lower $\alpha$. This was certainly the result of a lower amount of charges at lower $\alpha$, causing less repulsion. ${ }^{20}$ It must also be mentioned that the presence of salt - needed for the LS analysis - had a huge impact on the colloidal stability. Indeed, the macroRAFT agent prepared at $\alpha=7 \%$ could not be analyzed by LS at concentrations higher than $16 \mathrm{~g} \mathrm{~L}^{-1}$ due to the formation of a precipitate, whereas solutions of the $\mathrm{C}_{12}$-macroRAFT agent at $\alpha \geq 28 \%$ remained transparent up to $70 \mathrm{~g} \mathrm{~L}^{-1}$ at the same $\mathrm{NaCl}$ concentration.

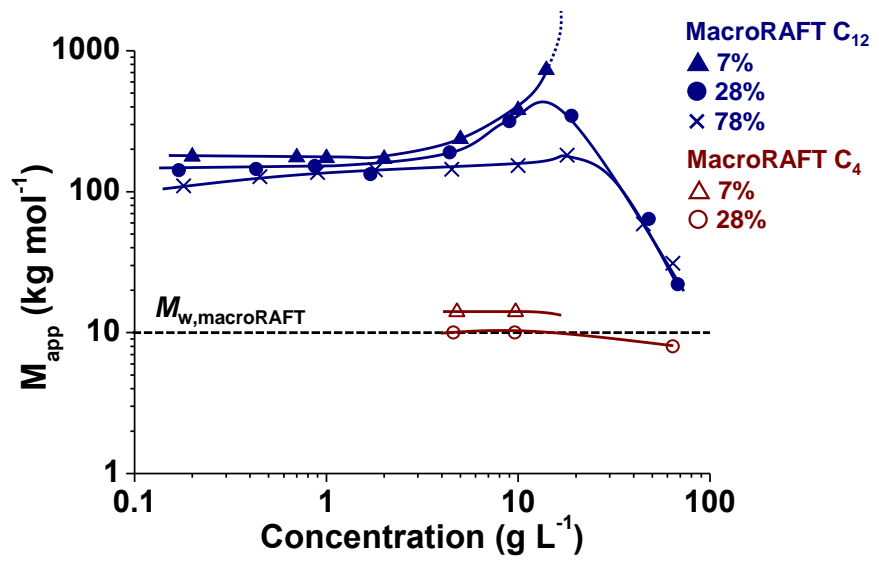

Figure 3. Evolution of the apparent molar mass $\mathrm{M}_{\text {app }}$ of $\mathrm{C}_{12}$-PDMAc-bis at $\alpha=7 \%(\mathbf{\Delta}), 28 \%(\boldsymbol{\bullet})$ and $78 \%(\times)$ and of $\mathrm{C}_{4}$-PDMAc at $\alpha=7 \%(\triangle)$ and $28 \%(\bigcirc)$ as a function of the polymer concentration obtained by light scattering at $20{ }^{\circ} \mathrm{C}$ in water. The solid lines are guide for the eyes. 
Dotted lines indicate precipitation of the sample caused by the addition of salt for LS analysis $\left([\mathrm{NaCl}]=0.01 \mathrm{~mol} \mathrm{~L}^{-1}\right)$.

In contrast to the $\mathrm{C}_{12}$-macroRAFT agent, $\mathrm{BAB}$ triblock copolymers with $D P_{\mathrm{n} / \text { arm }}$ PDAAm $\sim 10$ were turbid in the presence of $[\mathrm{NaCl}]=0.01 \mathrm{~mol} \mathrm{~L}^{-1}$ above respectively $\mathrm{C}=10 \mathrm{~g} \mathrm{~L}^{-1}$ for $\alpha=28 \%$ (sample $\mathrm{C}_{12}-12-28 \%$ ) and $\mathrm{C}=20 \mathrm{~g} \mathrm{~L}^{-1}$ for $\alpha=45 \%$ (sample $\mathrm{C}_{12}-10-45 \%$ ) (Figure S11B). These observations 1) qualitatively suggest that attraction due to bridging of flower-like micelles increased with the growth of PDAAm outer blocks from the macroRAFT and 2) confirmed that higher $\alpha$ decreased attraction even for short BAB triblock copolymers. Polymers with $D P_{\mathrm{n} / \mathrm{arm}}$ PDAAm $=21$ or 33 were not under thermodynamic equilibrium as explained in the previous part and were therefore not investigated by LS as a function of $\alpha$.

The results presented in this section confirmed that the loss of colloidal stability during PISA was due to attraction, i.e the bridging of flower-like micelles, which resulted in phase separation. This phenomenon was favored by decreasing $\alpha$ of the macroRAFT agent unit, increasing the ionic strength, decreasing the macroRAFT agent concentration and, thereby increasing the length of the PDAAm blocks. The loss of colloidal stability induced by bridging seemed sufficiently strong at $\alpha$ $=7 \%$ to result in a loss of polymerization control. This might be due to a difference in local concentration of the macroRAFT agent combined with a low $\left[\mathrm{C}_{12} \text {-macroRAFT }\right]_{0} /[\mathrm{ACPA}]_{0}$ ratio leading to the generation of several populations of polymer chains. For $\alpha=28 \%$, control was lost without stirring at $20 \mathrm{~g} \mathrm{~L}^{-1}$, but was preserved under stirring, suggesting that the heterogeneity of the reaction medium was not critical at this $\alpha$ and could be alleviated by stirring.

\subsection{Comparison of the $C_{4}$ and $C_{12}$-macroRAFT agents}

In our previous study, ${ }^{13}$ we observed that $\mathrm{BAB}$ polymers synthesized in similar conditions using telechelic macroRAFT agents end-capped by either dodecyl or butyl alkyl chains (named $\mathrm{C}_{12-}$ macroRAFT agent vs. $\mathrm{C}_{4}$-macroRAFT agent, see Scheme 2) led to significant differences in colloidal stability and polymerization control. These differences were confirmed in the present study, where we performed unstirred PISA experiments with both macroRAFT agents. For $[\text { macroRAFT }]_{0}=70 \mathrm{~g} \mathrm{~L}^{-1}$ at $70{ }^{\circ} \mathrm{C}$ and $\alpha=28 \%$ or $45 \%$, the polymerizations in presence of $\mathrm{C}_{4}$ - 
macroRAFT led systematically to phase separation whereas the polymerization medium remained homogeneous when the $\mathrm{C}_{12}$-macroRAFT was used in the same conditions. The results are summarized in Figure 4 (for details see Tables 1, S3, S4 and Figure S13). At $\alpha=45 \%$ phase separation was observed for $D P_{\mathrm{n} / \mathrm{arm}}$ PDAAm $\geq 32$ (and persisted when the sample was cooled to room temperature). At lower $\alpha(28 \%)$, this phase separation starts for lower monomer conversions $(<$ $20 \%$, corresponding to $D P_{\mathrm{n} / \mathrm{arm}}$ PDAAm $=10$ ), and becomes more pronounced with increasing conversion (see Figure S13B and C). Remarkably, the phase separation observed during PISA for short $D P_{\mathrm{n}}\left(D P_{\mathrm{n}}=10\right.$, sample $\mathrm{C}_{4}-10-28 \%$ in Table $\left.\mathbf{S 4}\right)$ was thermo-reversible (phase-separated at $70^{\circ} \mathrm{C}$ and monophasic at room temperature).

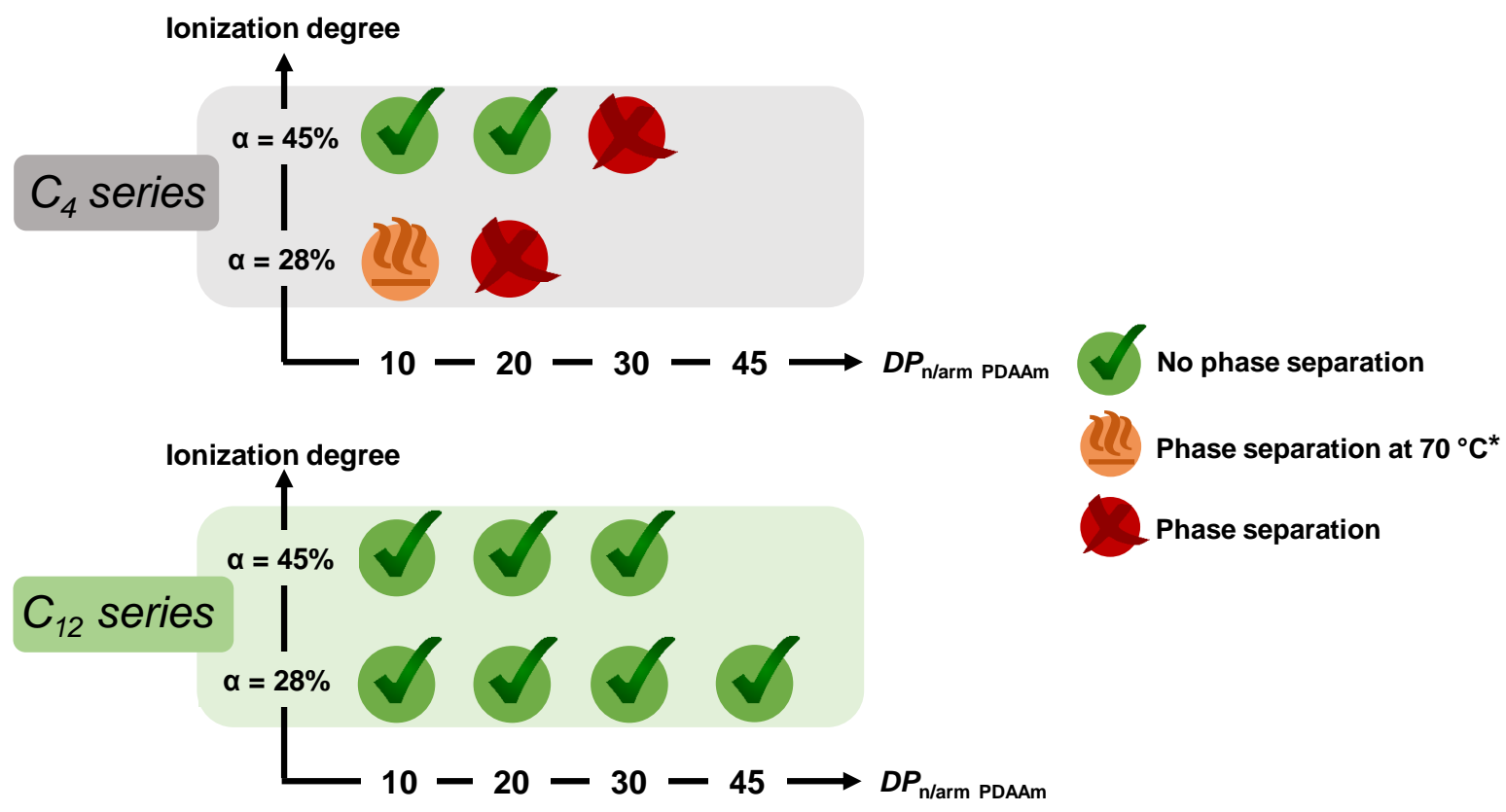

Figure 4. Summary of the macroscopic aspects of block copolymers dispersions synthesized with $10 \mathrm{wt} \%$ of DAAm without stirring either with $\mathrm{C}_{12}$ or $\mathrm{C}_{4}$-macroRAFT agent with [macroRAFT] $]_{0} \sim$ $70 \mathrm{~g} \mathrm{~L}^{-1}$ at $\alpha=28$ and $45 \%$. *Thermo-reversible phase separation observed: homogeneous sample at $25{ }^{\circ} \mathrm{C}$ that phase-separates reversibly at $70{ }^{\circ} \mathrm{C}$.

In order to understand the important divergences observed for the $\mathrm{C}_{4}$ - and the $\mathrm{C}_{12}$ - series, potential differences in the macromolecular chemical structure were first examined. For both series, the 
copolymers obtained by PISA were characterized by ${ }^{1} \mathrm{H}$ NMR and SEC at different monomer conversions, corresponding to $D P_{\mathrm{n} / \text { arm PDAAm }}$ of $\sim 10,20$ and 30 (for details see Table 1, S3, S4). Comparable $D P_{\mathrm{n} / \text { arm PDAAm }}$ were reached for both series, and SEC analyses revealed that the quality of control was similar with the $\mathrm{C}_{4}$ and the $\mathrm{C}_{12}$ macroRAFT, at least up to $D P_{\text {n/arm PDAAm }} \sim 30$ (see SEC in Figure S4, Figure S11A and Figure S12). Moreover, ${ }^{1} \mathrm{H}$ NMR analyses indicated that for both macroRAFT agents, the primary transfer reaction occurred on both trithiocarbonates of the macroRAFT agents quantitatively at very low monomer conversion $(<5 \%)$, leading to a bilateral polymer growth and the formation of triblock copolymers from the very beginning of the polymerization (see Figure S5 and explanatory text in the SI). In view of these results, the observed macroscopic differences for PISA series $\mathrm{C}_{4}$ and the $\mathrm{C}_{12}$ could not be explained by strong differences in the macromolecular chemical structure of the $\mathrm{BAB}$ triblock copolymers.

Potential differences in terms of supramolecular self-assembly were then investigated by LS experiments. At low concentration (Figure 3), an $\mathrm{M}_{\text {app }}$ around $10 \mathrm{~kg} \mathrm{~mol}^{-1}$ was determined for the $\mathrm{C}_{4}$-macroRAFT (independent of $\alpha$ ), which was very close to the molar mass of the macroRAFT agent determined by ${ }^{1} \mathrm{H}$ NMR $\left(M_{\mathrm{n}, \mathrm{NMR}}=8.9 \mathrm{~kg} \mathrm{~mol}^{-1}\right)$. These experiments revealed that, in contrast to the $\mathrm{C}_{12}$-macroRAFT agent, the $\mathrm{C}_{4}$-macroRAFT agent did not self-assemble in water. This conclusion was also confirmed by the difference in viscosity observed for both macroRAFT agents: a distinct increase of viscosity with polymer concentration could be observed for aqueous solutions of $\mathrm{C}_{12}$-macroRAFT agent, whereas no significant increase of viscosity could be observed for $\mathrm{C}_{4}$ macroRAFT agent, at least not by the naked eye. This decrease of association with the decrease of the alkyl length is consistent with the literature on telechelic homopolymers decorated on both sides with alkyl chains. ${ }^{21}$ These results show that, unlike the $\mathrm{C}_{12}$-system, the RAFT dispersion polymerization of DAAm with the $\mathrm{C}_{4}$-macroRAFT agent does not start in a percolating transient network, but in a non-associative system.

Block copolymers of $D P_{\mathrm{n} / \text { arm PDAAm }} \sim 10$ synthesized with both macroRAFT agents in comparable conditions ( $\alpha=45 \%, \mathrm{C}_{4}$-macroRAFT: entry $\mathrm{C}_{4}-11-45 \%$ in Table S4, and $\mathrm{C}_{12}$-macroRAFT: entry $\mathrm{C}_{12}-10-45 \%$ in Table S3) were then investigated by LS analyses in order to understand how the assembly evolved upon the growth of the PDAAm side chains (see Figure 5). At low concentrations $\left(C<10 \mathrm{~g} \mathrm{~L}^{-1}\right), \mathrm{M}_{\text {app }}$ was significantly smaller for the $\mathrm{C}_{4}$ series $\left(\mathrm{C}_{4}-11-45 \%\right)$ than for the $\mathrm{C}_{12}$ series $\left(\mathrm{C}_{12}-10-45 \%\right)$; but a progressive increase of aggregation with the concentration 
was observed for the $\mathrm{C}_{4}$-system. $\mathrm{M}_{\text {app }}$ and $\mathrm{R}_{\mathrm{h} \text {,app }}$ became similar for both polymers at $\mathrm{C}=20 \mathrm{~g} \mathrm{~L}^{-1}$, implying that they both formed flower-like micelles with a similar extent of aggregation in spite of a seemingly weaker cooperativity of the self-assembly for $\mathrm{C}_{4}-11-45 \%$. Unfortunately, it was not possible to follow the evolution of $\mathrm{M}_{\text {app }}$ at concentrations higher than 20-30 $\mathrm{g} \mathrm{L}^{-1}$ (which could have given clearer information on attraction) because the addition of $0.01 \mathrm{M} \mathrm{NaCl}$ for the LS studies caused significant turbidity. Polymers with $D P_{\text {n/arm PDAAm }}=20$ or 30 were not under thermodynamic equilibrium, as explained in the previous parts, and were therefore not investigated either. Overall, the LS experiments indicated that the initial state of the macroRAFT agents was very different for the $\mathrm{C}_{4}$ and the $\mathrm{C}_{12}$ macroRAFT at $\alpha=45 \%$, but that flower-like micelles with similar extent of aggregation were obtained as soon as the PDAAm arms reached a $\mathrm{DP}_{\mathrm{n} / \text { arm PDAAm }}$ $\sim 10$.

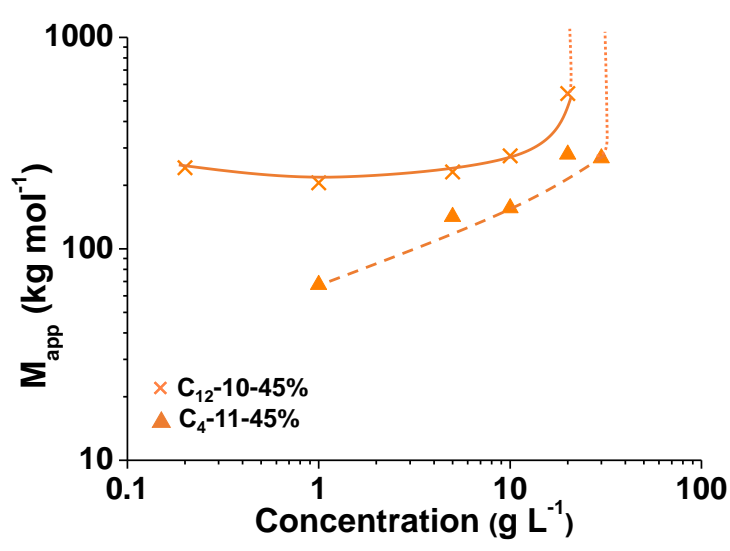

Figure 5. Evolution of the apparent molar mass $\mathrm{M}_{\mathrm{app}}$ as a function of the polymer concentration obtained by light scattering at $20^{\circ} \mathrm{C}$ in water for block copolymers with $D P_{\mathrm{n} / \mathrm{arm}} \sim 10$, prepared by dispersion polymerization of DAAm in $\mathrm{D}_{2} \mathrm{O}$ at $70{ }^{\circ} \mathrm{C}$ with the $\mathrm{C}_{12}$-macroRAFT agent (see Table S3) or $\mathrm{C}_{4}$-macroRAFT agent (see Table S4; the scattered intensity was too low below $1 \mathrm{~g} \mathrm{~L}^{-1}$ to obtain reliable data) at $\alpha=45 \%$ without stirring. The solid lines are guide for the eyes. Dotted lines indicate precipitation of the sample caused by the addition of salt for LS analysis $([\mathrm{NaCl}]=0.01$ $\left.\mathrm{mol} \mathrm{L} \mathrm{L}^{-1}\right)$.

To summarize, the results suggest that the growth of the B blocks occurred symmetrically on both sides of the macroRAFT agents for both series of polymers. The $\mathrm{C}_{4}$-macroRAFT was not self- 
assembled initially, contrary to the $\mathrm{C}_{12}$ one, but flower-like micelles of similar extent of aggregation and dimensions were observed for both series of BAB triblock copolymers with $D P_{\mathrm{n} / \mathrm{arm}}$ PDAAm $~$ 10. This implied that both series of polymers form a percolated network of bridged flower-like micelles starting from $D P_{\mathrm{n} / \text { arm }}$ PDAAm $=10$. From this, it was deduced that the phase separation observed at sufficiently high $D P_{\mathrm{n} / \mathrm{arm}}$ PDAAm for the $\mathrm{C}_{4}$-series (at both $\alpha=28 \%$ or $45 \%$, see Figure 4) was caused by a strong attraction between bridged micelles. In contrast, for the $\mathrm{C}_{12}$-series no phase separation was observed in these conditions ([macroRAFT $]_{0}=70 \mathrm{~g} \mathrm{~L}^{-1}$ ), but only for lower macroRAFT concentrations, i.e. for higher $D P_{\mathrm{n} / \mathrm{arm}}$ PDAAm, and at low $\alpha(28 \%)$, as described in part 2.1. Finally, we explain the thermo-sensitive phase-separation observed for $\mathrm{C}_{4}-10-28 \%$ (no phase separation at $20^{\circ} \mathrm{C}$, phase separation at $70^{\circ} \mathrm{C}$ ) by the thermo-sensitive behavior of the PDAAm blocks which has been reported before in the litterature. ${ }^{35-37}$

The fact that obvious differences between the $\mathrm{C}_{4}$ and the $\mathrm{C}_{12}$-series were observed in terms of phase separation for $D P_{\mathrm{n} / \mathrm{arm}}$ PDAAm $~ 10$ (see Figure 4) implies that these differences must have a thermodynamic origin. Indeed, the light scattering experiments presented in part 1 for sample $\mathrm{C}_{12}$ $10-28 \%$ and the fact that the thermally-induced phase separation observed for sample $\mathrm{C}_{4}-10-28 \%$ is reversible indicated that both series of polymers with a $D P_{\mathrm{n} / \mathrm{arm}}$ PDAAm $\sim 10$ are at thermodynamic equilibrium. From the observations we must conclude that the attraction, i.e. the propensity to form bridges rather than loops, was stronger for the $\mathrm{C}_{4}$-series than for the $\mathrm{C}_{12}$-series. One potential origin for the difference of attraction in both series might be the presence of a small but distinct amount of monofunctional polymers ( $\mathrm{AB}$ diblocks) in the $\mathrm{BAB}$ triblocks. It has been reported in the literature that a small fraction of $\mathrm{AB}$ copolymers in $\mathrm{BAB}$ triblock copolymers reduces attraction. ${ }^{21}$ The formation of a small quantity of $\mathrm{AB}$ diblock copolymers is actually inherent to the RAFT mechanism, but as $\mathrm{C}_{4}$ and $\mathrm{C}_{12}$ series were performed in identical conditions, we may reasonably assume that the observed differences should result from monofunctional RAFT agent present in different quantities in the $\mathrm{C}_{4}$ and $\mathrm{C}_{12}$ molecular RAFT agent, $\left(\mathrm{C}_{12} \text {-TTC-PDMAc) }\right)_{2}$-BA and $\left(\mathrm{C}_{4}\right.$ TTC-PDMAc)2-BA). Indeed, the SEC chromatograms of the $\mathrm{C}_{12}$-series (see Figures S4 and S11) do exhibit a more pronounced shoulder in the lower molar mass region than those prepared from the $\mathrm{C}_{4}$-macroRAFT (Figure S12). This suggests the presence of a slightly larger amount of $\mathrm{AB}$ diblocks in the $\mathrm{C}_{12}$ than in the $\mathrm{C}_{4}$ series and could explain a somewhat lower attraction for the former. We also note that for $D P_{\mathrm{n} / \text { arm }}$ PDAAm $\geq 20$, the exchange of $\mathrm{B}$ blocks between micelles becomes extremely slow and the assemblies become frozen. The differences between the $\mathrm{C}_{4}$ and 
the $\mathrm{C}_{12}$-series may therefore not only have a thermodynamic but also a kinetic origin: it is possible that the $\mathrm{C}_{4}$-series, which starts from an unassembled state for $D P_{\mathrm{n} / \mathrm{arm}}$ PDAAm $=0\left(\mathrm{C}_{4}\right.$-macroRAFT agent), does not have time to rearrange in a homogeneous transient network during polymerization before the exchange rate of PDAAm blocks becomes too slow. This would then result in a different organization compared to the $\mathrm{C}_{12}$-series and potentially in a different ratio of bridges over loops favoring phase separation in the $\mathrm{C}_{4}$ series.

\section{Conclusion}

In this paper, we investigated by light scattering and macroscopic observations the behavior of BAB triblock copolymers prepared by PISA in dispersion in order to understand the main features of these complex polymerizations.

The bifunctional $\mathrm{C}_{12}$-macroRAFT agent was clearly shown to self-assemble into a dynamic percolated network of flower-like micelles at the beginning of the polymerization. Upon polymerization, the growth of the insoluble PDAAm blocks caused a decrease of the exchange rate of the bridges between the micelles until the network eventually became frozen. This occurred for a $D P_{\mathrm{n} / \text { arm PDAAm }}$ ranging between 10 and 20. As a consequence, synthesis of these BAB triblocks under stirring resulted in an irreversible breaking of the network into tiny particles consisting of a few bridged flower-like micelles, whereas synthesis without stirring afforded a non-flowing gel. The bifunctional $\mathrm{C}_{4}$-macroRAFT agent was initially not self-assembled, but also formed a transient network of bridged flower-like micelles upon growth of the PDAAm blocks.

A second consequence of the synthesis of BAB triblock copolymers instead of $\mathrm{AB}$ diblock copolymers was the fact that bridging between flower-like micelles could result into macroscopic phase separation because of the formation of intermicellar bridges instead of intramicellar loops. This loss of colloidal stability could be accompanied with a loss of polymerization control. Attraction caused by bridging of flower-like micelles, resulting eventually in phase separation, was favored by increase of ionic strength, growth of the PDAAm arms, decrease of macroRAFT agent concentration or ionization degree $(\alpha)$. This study explains why it was not possible to obtain colloidally stable dispersions and controlled polymerizations using a neutral bifunctional macroRAFT agent. ${ }^{13}$ It was also observed that replacement of the $\mathrm{C}_{12}$ alkyl chains by $\mathrm{C}_{4}$ ones 
favored phase separation. This might be due to the presence of a smaller amount of monofunctional RAFT agents in the $\mathrm{C}_{4}$-series limiting the quantity of $\mathrm{AB}$ diblock copolymers present in the sample. In contrast, the absence of phase separation for the $\mathrm{C}_{12}$-series could be explained by the presence of a larger amount of $\mathrm{AB}$ diblock copolymers, limiting attraction. We believe that this study gives a general picture of the mechanism of assembly of BAB triblock copolymers during PISA and proposes clues to achieve colloidal stability and control during the polymerization.

Acknowledgement: The authors thank Jean-Michel Guigner (IMPMC, Sorbonne Université) for cryo-TEM analyses and Taco Nicolai (IMMM, Le Mans Université) for scientific discussions. The authors acknowledge the Agence Nationale de la Recherche for the following research grants: PISAForFilms project (ANR-17-CE09-0031-01) and Dynamic-PISA (ANR-19-CE06-0002-01).

Keywords: PISA, aqueous RAFT dispersion polymerization, BAB triblock copolymers, flowerlike micelles, transient network, light scattering

\section{References}

1 W. Wang, W. Lu, A. Goodwin, H. Wang, P. Yin, N.-G. Kang, K. Hong and J. W. Mays, Progress in Polymer Science, 2019, 95, 1-31.

2 P. Tyagi, A. Deratani, D. Bouyer, D. Cot, V. Gence, M. Barboiu, T. N. T. Phan, D. Bertin, D. Gigmes and D. Quemener, Angewandte Chemie International Edition, 2012, 51, 7166-7170.

3 B. Baroli, Journal of Pharmaceutical Sciences, 2007, 96, 2197-2223.

4 C. Tsitsilianis, Soft Matter, 2010, 6, 2372.

5 B. Charleux, G. Delaittre, J. Rieger and F. D'Agosto, Macromolecules, 2012, 45, 6753-6765.

6 J.-T. Sun, C.-Y. Hong and C.-Y. Pan, Polymer Chemistry, 2013, 4, 873-881.

7 J. Rieger, Macromolecular Rapid Communications, 2015, 36, 1458-1471.

8 M. J. Derry, L. A. Fielding and S. P. Armes, Progress in Polymer Science, 2016, 52, 1-18.

9 C. Gao, S. Li, Q. Li, P. Shi, S. A. Shah and W. Zhang, Polym. Chem., 2014, 5, 6957-6966.

10 C. Gao, J. Wu, H. Zhou, Y. Qu, B. Li and W. Zhang, Macromolecules, 2016, 49, 4490-4500.

11 Y. Qu, S. Wang, H. Khan, C. Gao, H. Zhou and W. Zhang, Polymer Chemistry, 2016, 7, 1953-1962.

12 W. Wang, C. Gao, Y. Qu, Z. Song and W. Zhang, Macromolecules, 2016, 49, 2772-2781.

13 P. Biais, P. Beaunier, F. Stoffelbach and J. Rieger, Polymer Chemistry, 2018, 9, 4483-4491.

14 C. Grazon, J. Rieger, N. Sanson and B. Charleux, Soft Matter, 2011, 7, 3482.

15 W. Zhang, F. D’Agosto, O. Boyron, J. Rieger and B. Charleux, Macromolecules, 2012, 45, 4075-4084.

16 E. R. Jones, O. O. Mykhaylyk, M. Semsarilar, M. Boerakker, P. Wyman and S. P. Armes, Macromolecules, 2016, 49, 172-181.

17 F. Brunel, J. Lesage de la Haye, M. Lansalot and F. D’Agosto, J. Phys. Chem. B, 2019, 123, 66096617.

18 C. Chassenieux, T. Nicolai and L. Benyahia, Current Opinion in Colloid \& Interface Science, 2011, 16, $18-26$.

19 T. Annable, R. Buscall, R. Ettelaie and D. Whittlestone, Journal of Rheology, 1993, 37, 695-726. 
20 C. Charbonneau, M. M. De Souza Lima, C. Chassenieux, O. Colombani and T. Nicolai, Physical Chemistry Chemical Physics, 2013, 15, 3955.

21 F. Laflèche, T. Nicolai, D. Durand, Y. Gnanou and D. Taton, Macromolecules, 2003, 36, 1341-1348.

22 A. N. Semenov, J.-F. Joanny and A. R. Khokhlov, Macromolecules, 1995, 28, 1066-1075.

23 Q. T. Pham, W. B. Russel, J. C. Thibeault and W. Lau, Macromolecules, 1999, 32, 2996-3005.

24 F. Laflèche, D. Durand and T. Nicolai, Macromolecules, 2003, 36, 1331-1340.

25 T. Nicolai, O. Colombani and C. Chassenieux, Soft Matter, 2010, 6, 3111.

26 A. Halperin and S. Alexander, Macromolecules, 1989, 22, 2403-2412.

27 J. Rieger, F. Stoffelbach, C. Bui, D. Alaimo, C. Jérôme and B. Charleux, Macromolecules, 2008, 41, 4065-4068.

28 G. G. Cameron, Polymer International, 1995, 38, 106-106.

29 S. Michielsen, in Polymer Handbook, John Wiley \& Sons, Inc., New York, 4. ed., 1999, p. VII/547627.

30 W. Brown, Dynamic Light Scattering: The Method and Some Applications, Oxford University Press, New York, 1993.

31 J. P. Patterson, M. P. Robin, C. Chassenieux, O. Colombani and R. K. O'Reilly, Chem. Soc. Rev., 2014, 43, 2412-2425.

32 J. Lesage de La Haye, J.-M. Guigner, E. Marceau, L. Ruhlmann, B. Hasenknopf, E. Lacôte and J. Rieger, Chemistry - A European Journal, 2015, 21, 2948-2953.

33 J. Dubochet, M. Adrian, J.-J. Chang, J.-C. Homo, J. Lepault, A. W. McDowall and P. Schultz, Quarterly Reviews of Biophysics, 1988, 21, 129.

34 C. Chassenieux, T. Nicolai and D. Durand, Macromolecules, 1997, 30, 4952-4958.

35 S. J. Byard, M. Williams, B. E. McKenzie, A. Blanazs and S. P. Armes, Macromolecules, 2017, 50, 1482-1493.

36 G. Mellot, P. Beaunier, J.-M. Guigner, L. Bouteiller, J. Rieger and F. Stoffelbach, Macromolecular Rapid Communications, 2018, 40, 1800315.

37 X. Wang, J. Zhou, X. Lv, B. Zhang and Z. An, Macromolecules, 2017, 50, 7222-7232. 